Document downloaded from:

http://hdl.handle.net/10251/103723

This paper must be cited as:

Montes-Robles, R.; Hernández-Montoto, A.; Ibáñez Civera, FJ.; Masot Peris, R.; De La Torre-Paredes, C.; Martínez-Máñez, R.; Garcia-Breijo, E.... (2017). Design of a low-cost equipment for optical hyperthermia. Sensors and Actuators A Physical. 255:61-70. doi:10.1016/j.sna.2016.12.018

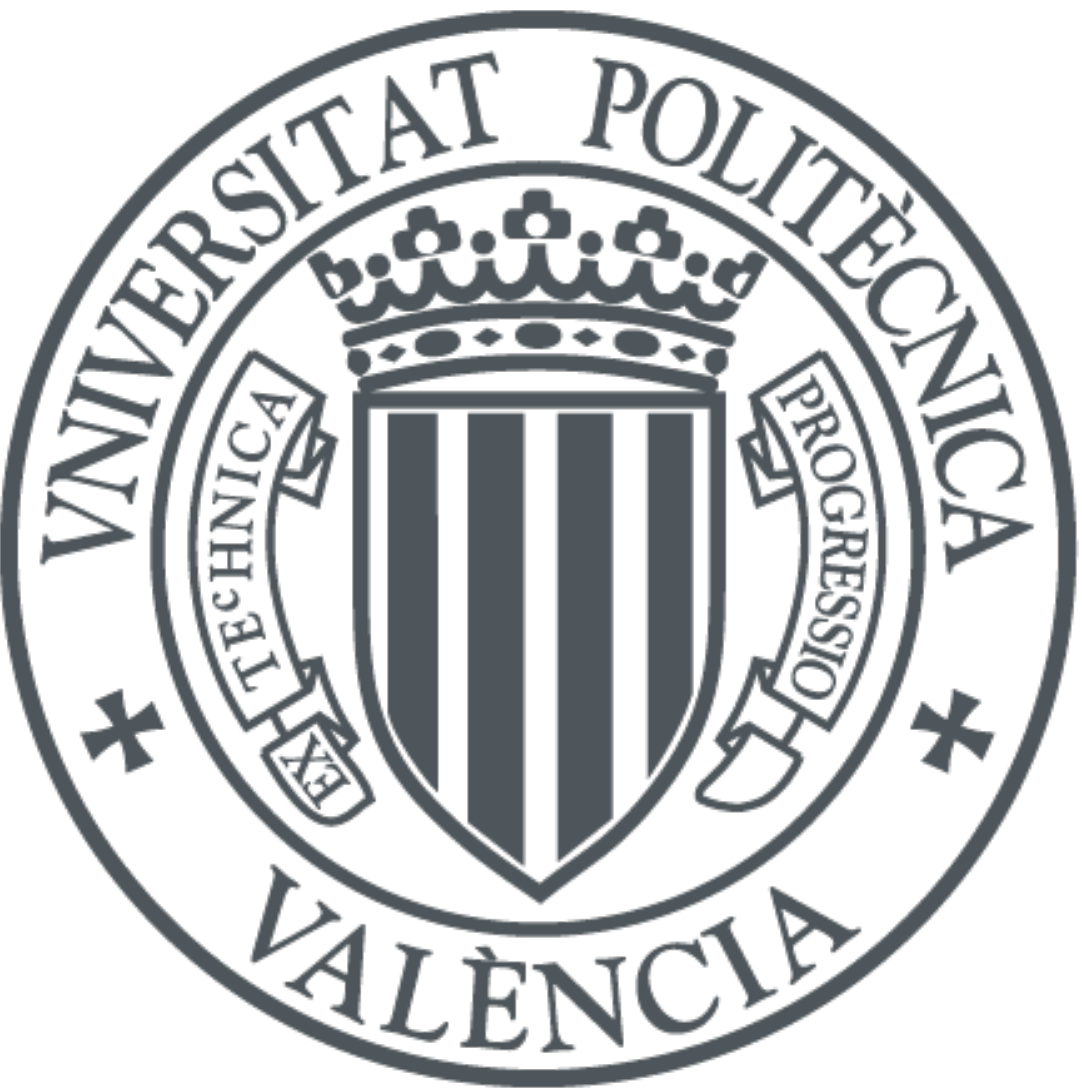

The final publication is available at

http://doi.org/10.1016/j.sna.2016.12.018

Copyright Elsevier

Additional Information 
This is a pre- edited version of:

Roberto Montes-Robles, Andy Hernández, Javier Ibáñez, Rafael Masot-Peris, Cristina de la Torre, Ramón Martínez-Máñez, Eduardo García-Breijo, Rubén Fraile, Design of a low-cost equipment for optical hyperthermia, Sensors and Actuators A: Physical, Volume 255, 1 March 2017, Pages 61-70

The full paper in its final form can be found at: http://www.sciencedirect.com/science/article/pii/

s0924424716311840

\title{
Design of a low-cost equipment for optical hyperthermia
}

\author{
Roberto Montes-Robles ${ }^{\mathrm{a}}$, Andy Hernández ${ }^{\mathrm{a}}$, Javier Ibáñez ${ }^{\mathrm{a}}$, Rafael \\ Masot-Peris $^{\mathrm{a}}$, Cristina de la Torre ${ }^{\mathrm{a}}$, Ramón Martínez-Máñez ${ }^{\mathrm{a}, \mathrm{b}}$, Eduardo \\ García-Breijo $^{a}$, Rubén Fraile ${ }^{c}$ \\ ${ }^{a}$ Instituto Interuniversitario de Reconocimiento Molecular y Desarrollo Tecnológico \\ (IDM). Universitat Politècnica de València - Universitat de València. \\ Camino de Vera s/n. 46022 Valencia, Spain. \\ ${ }^{b}$ CIBER de Bioingeniería, Biomateriales y Nanomedicina (CIBER-BBN). \\ ${ }^{c}$ Centro de Investigación en Tecnologías Software y Sistemas Multimedia para la \\ Sostenibilidad (CITSEM). \\ Universidad Politécnica de Madrid. Campus Sur. \\ Edificio La Arboleda. Calle Alan Turing 3. \\ 28031 Madrid, Spain.
}

\begin{abstract}
A laser equipment is presented which has been specifically designed for optical hyperthermia. Such specificity in the design has allowed implementation at costs significantly lower than other options currently available in the market. The developed equipment includes a $808 \mathrm{~nm}$ laser whose output power is up to $500 \mathrm{~mW}$. It also incorporates additional devices that help the user in calibrating the system and supervising it while functioning. The performance of a prototype is tested by running two hyperthermia experiments: one involving gold nanorods, and the other using gold nanostars. The specific set-up of this prototype has allowed laser irradiation with radiated power densities up to $4 \mathrm{~W} / \mathrm{cm}^{2}$.
\end{abstract}

Keywords: Laser applications, Gold nanoparticles, Hyperthermia equipment

\section{Introduction}

Humankind has been fighting against cancer for decades. Presently, interdisciplinary cooperation between experts in medicine, bioengineering, electronics and nanotechnology is being key in achieving advances in such battle. Optical hyperthermia is an exemplary outcome of such cooperation. 
This technology aims at causing the death to tumour cells by overheating them. Overheating is achieved by irradiating specifically synthesised metallic nanoparticles (size below $100 \mathrm{~nm}$ ) with coherent electromagnetic waves [1]. On receiving the radiation, these particles are heated and act themselves as heaters for the tumour cells. Among all metallic nanoparticles, gold nanoparticles (Au-NPs) have photothermic properties [2] that make them especially adequate for this type of cancer treatment [3].

When Au-NPs are irradiated by a light source at a certain wavelength, they heat up as a result of electronic oscillation. This effect is known as surface plasmon resonance [4] and it makes the Au-NPs act as efficient energy converters $[5,6,7]$ that receive light energy and convert it to heat. Afterwards, they deliver such heat to their closest surroundings [8, 9]. Good penetration of irradiated electromagnetic waves into cell tissues can be achieved with wavelengths in the near infra-red range (NIR), that is, between $650 \mathrm{~nm}$ and $900 \mathrm{~nm}$ [10]. This is due to the cell tissues absorbing very little energy in the NIR [11]. Conversely, the morphology of the nanoparticles should be preferably chosen so that their absorption is maximised at the NIR. One of such morphologies well-fitted to the NIR is nanorod, since nanorods can be grown in a controlled fashion to achieve longitudinal absorption near $800 \mathrm{~nm}$ $[12,13]$. Nanostar is an alternative morphology, in which plasmonic properties are enhanced by star tips $[14,15]$.

Metallic nanoparticles are heated up to temperatures between $40^{\circ} \mathrm{C}$ and $48^{\circ} \mathrm{C}$ for cancer therapy applications [16]. Due to their specific vascular system structure, tumour cells are much more sensitive to high temperatures that healthy cells. At this temperature range, the cell membrane and the cytoskeleton of tumour cells begin to degrade. In addition, if high temperatures are maintained along time, protein denaturation happens in the cytoplasm. Such denaturation affects heat shock proteins (HSP), so the tumour cell becomes more vulnerable to hyperthermia as its exposure to high temperatures is prolonged [17]. Last, the inefficiency in the vascular system affects the delivery of oxygen and nutrients throughout the cell, hence making cell recovery after hyperthermia more difficult. Therefore, optical hyperthermia does not cause cell death directly; instead, it generates the conditions in which tumour cells degenerate and, as a result, die. In this sense, the process is analogue to programmed cell death or apoptosis. In contrast to other thermal treatments such as thermal ablation or carbonisation, apoptosis-like processes have the advantage of avoiding the side effect of inflammation, which may evolve into either sepsis or gangrene [18]. 
When compared to another hyperthermia technology as magnetic hyperthermia, optical hyperthermia has the advantage of requiring lower loadings of metallic nanoparticles at the target tissues [19]. Additionally, in optical hyperthermia nanoparticle heating only happens in tissue areas that fall inside the laser beam. This makes it possible to heat only specific cells. In contrast, magnetic hyperthermia can only be applied at bulk tissue level, which implies the destruction of normal non-malignant cells [19, 20]. The main limitation of optical hyperthermia is that it can only be applied to superficial lesions, due to the limited penetration of optical radiation into tissues [20].

Beyond hyperthermia, metallic nanoparticles have many other applications due to their properties [21], including biocompatibility [22]. For instance, nanoparticles are used as contrast agents in magnetic resonance imaging (MRI). They can also be applied in controlled drug release [23] since they can be easily functionalised by a wide range of ligands, including antibodies, polymers, genetic material, and so on [24, 25, 26].

There currently exist in the market several systems incorporating laser actuators that can be used for running experiments involving optical hyperthermia. However, usually such equipments are expensive. Since they are designed for general use in laser applications, their capabilities tend to be over-dimensioned for optical hyperthermia. In this paper, a laser equipment is presented which has been specifically designed for optical hyperthermia and controlled drug release. Such specificity has allowed to exclude from the design some parts of generic equipments that are superfluous for the target application. As a result, a lower-cost equipment has been developed that includes a $808 \mathrm{~nm}$ laser [27] whose output power is up to $500 \mathrm{~mW}$, an electronic microscope that helps in calibrating the radiated power density and a thermostatic chamber that serves the purpose of controlling the ambient conditions of the experiments. Equipment function is tested by running two hyperthermia experiments, one involving gold nanorods, and the other using gold nanostars.

\section{Hardware description}

\subsection{Overview}

The designed equipment (see Figure 1) has two different parts. On the one hand, the power unit has the function of providing the laser diode with the required electrical current. The current source is voltage-controlled by a 

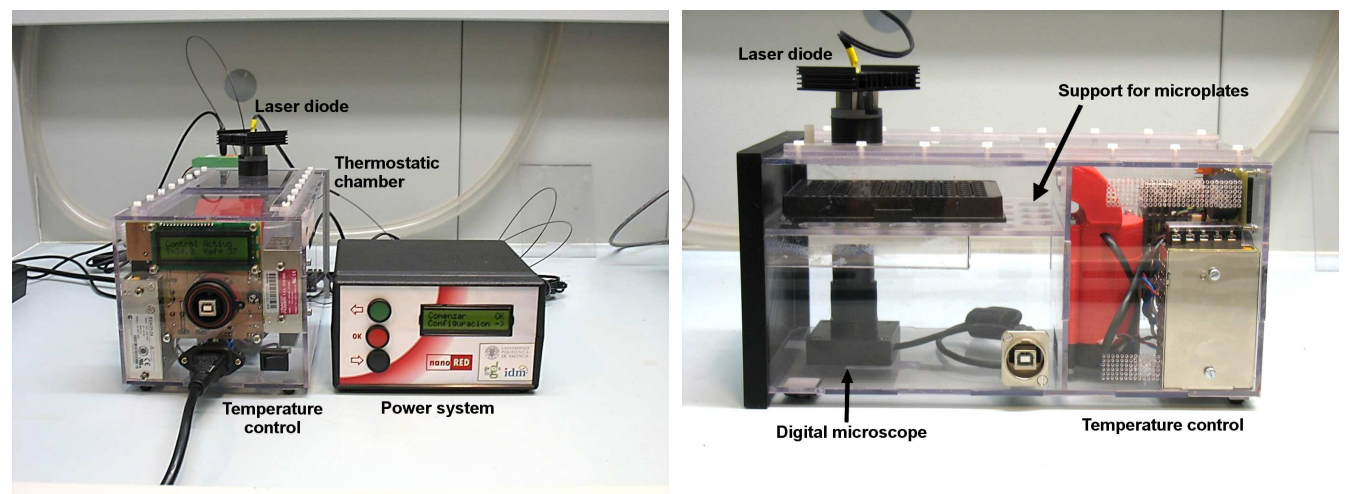

Figure 1: Frontal and lateral views of the developed system.

8-bit microcontroller (PIC18F2550, manufactured by Microchip Technology Inc). A button panel and a $2 \times 16$ character display form the user interface of this power unit. On the other hand, the optical system includes the aforementioned laser diode and a vision subsystem whose function is to estimate the area of the light spot. Figure 2 shows a block diagram of the whole equipment.

Additionally, the developed equipment includes a thermostatic chamber (Figure 1, right). This chamber has been specifically designed so that it can host all the rest of elements (optical system, power system, samples to be heated, etc.) in the desired positions. It includes a controller so that the temperature inside it can be controlled by a human operator.

The following subsections include more detailed descriptions of each one of these elements: optical system, power unit, and thermostatic chamber.

\subsection{Optical system}

The optical system is the key element in the designed equipment, since its specifications set the requirements for the power unit. The main function of the optical system is the emission of an infrared monomode light having a given power per unit surface. Such power density should be big enough for the light beam to excite the suspended Au-NPs. The magnitude of the radiated power density mainly depends on the output power of the laser diode and the focus adjustment. 


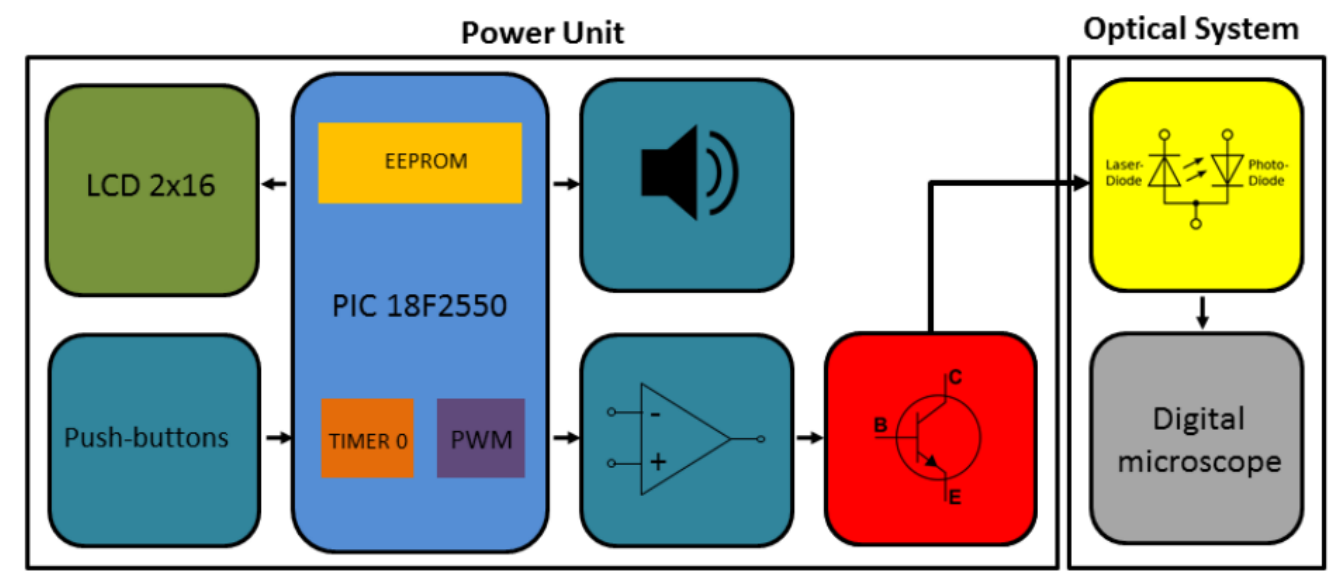

Figure 2: Block diagram.

\subsubsection{Laser emission}

The core of the laser emitting block (upper right box in Figure 2) is a LDM-0808-500m-92 laser diode, manufactured by Roithner Lasertechnik $G m b H$. Nominal wavelength of the radiated laser is equal to $808 \pm 10 \mathrm{~nm}$ (infrared range), and nominal power equal to $500 \mathrm{~mW}$. By definition, laser light is coherent. Laser diodes achieve this coherence by limiting oscillations to a single mode. In turn, the divergence of the emitted beam is large. Specifically, for this diode the beam divergence is around 40 degrees at the normal plane $\left(\theta_{\perp}=40^{\circ}\right)$ and 10 degrees at the parallel plane $\left(\theta_{\|}=10^{\circ}\right)$. This results on the laser beam having the shape of an elliptical cone with growing cross section.

Beam divergence has been reduced by placing a collimator (Roithner Lasertechnik RLY-133G) at the output of the laser diode. This collimator comprises three glass lenses mounted on a threaded surface $(\mathrm{M} 9 \times 0.5$ $\mathrm{mm})$. This allows a fine adjustment of the diode-lens distance. The collimator housing is cylindrical, with $13 \mathrm{~mm}$ height and $9 \mathrm{~mm}$ diameter. The focal distance equals $8.2 \mathrm{~mm}$. Lenses are coated to avoid reflections in the infrared range.

The collimator opening is $5 \mathrm{~mm}$ width. As a consequence, the maximum distance between collimator lens and diode in order to minimise the power 


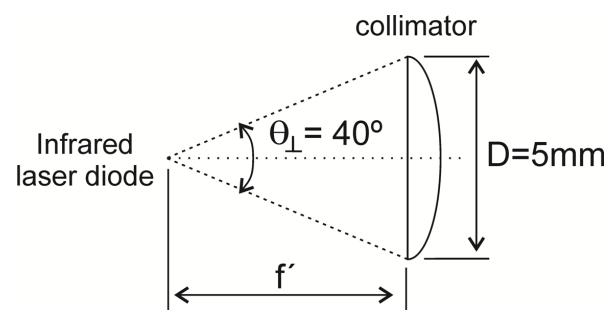

Figure 3: Diagram of collimator and focal distance.

radiated out of the lens is (see Figure 3):

$$
f_{\mathrm{MAX}}=\frac{D}{2} \frac{1}{\tan \left(\theta_{\perp} / 2\right)}
$$

where $D$ is the lens opening diameter. Since $D=5 \mathrm{~mm}$ and $\theta_{\perp}=40^{\circ}$, it results $f_{\mathrm{MAX}}=6.86 \mathrm{~mm}$.

\subsubsection{Vision system}

The vision system plays a relevant role in the calibration of the laser device. Specifically, it serves the purpose of estimating the surface density of the radiated power, since it allows measuring the area of the laser spot. Its basic working procedure is as follows. The laser beam, after going through the collimator, reaches a white translucent surface from the top. This surface is placed at a distance from the collimator lenses equal to that of the target substance container in the on-line working set-up. The bottom side of this surface is marked with a calibrated grid whose square divisions are numbered (Figure 4).

On reaching the translucent surface, the laser beam produces a elliptical spot that is visible from below the surface. This spot, with the calibrated grid superimposed on it, is captured by a digital microscope (Cacolms, manufactured by Velleman $n v$ ) connected to a standard personal computer (PC). The resulting photographic image sent to the $\mathrm{PC}$ is used to calculate the surface of the illuminated spot. Since the shape is elliptical, its surface $S$ can be calculated after measuring the two main axes of the ellipse:

$$
S=\pi \cdot a \cdot b
$$

where $a$ and $b$ are the lengths of the semiaxes of the ellipse (Figure 4). From this calculation, the radiated power density of the laser can be estimated as:

$$
L=\frac{P_{\text {laser }}}{S}=\frac{P_{\text {laser }}}{\pi a b}
$$




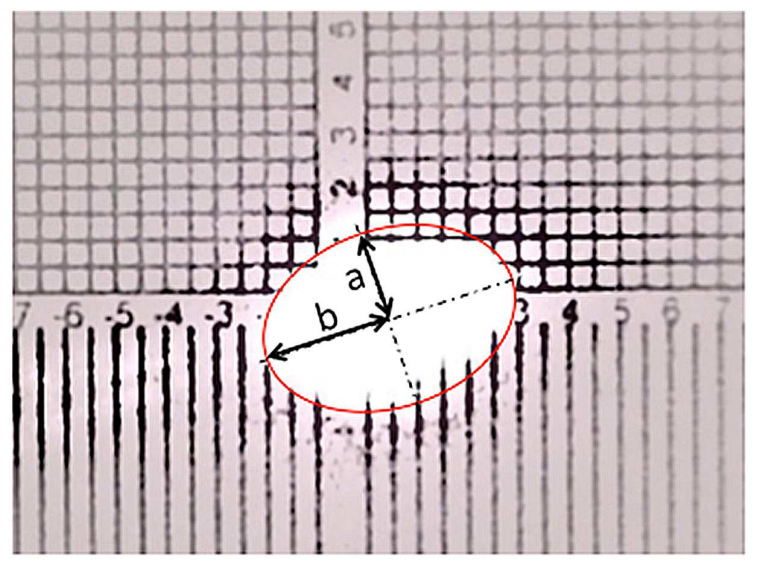

Figure 4: Calibrated grid $(1$ division $=0.5 \mathrm{~mm})$ at the bottom side of the translucent surface.

Particularly, for the nominal power of the utilised laser diode $\left(P_{\text {laser }}=\right.$ $500 \mathrm{~mW}$ ) the resulting maximum radiated power density for our system set-up was $L=4.24 \mathrm{~W} / \mathrm{cm}^{2}$.

\subsection{Power system}

The power system mainly consists of two blocks: a voltage-to-current converter (the two boxes at the lower-right end of the power unit in Figure 2) and a controller (the rest of boxes of the power unit in Figure 2). The laser diode is fed with current by a voltage-to-current converter, which includes: a voltage follower, a low-pass filter, a transadmittance amplifier, and a current amplifier. This converter is controlled by a pulse-width modulated (PWM) signal generated by the controller.

\subsubsection{Voltage-to-current converter}

The electronic schema of the voltage-to-current converter is depicted in Figure 5. The input PWM voltage signal first goes through a voltage follower and a low-pass RLC filter that acts as an integrator. These convert it into a voltage signal $V_{1}$ whose amplitude is proportional to the pulse width of the PWM input. The joint effect of the transadmittance and current amplifiers is to generate a current proportional to $V_{1}$ which is delivered to the laser diode. The system is designed to provide output currents ranging from 200 to $650 \mathrm{~mA}$. 


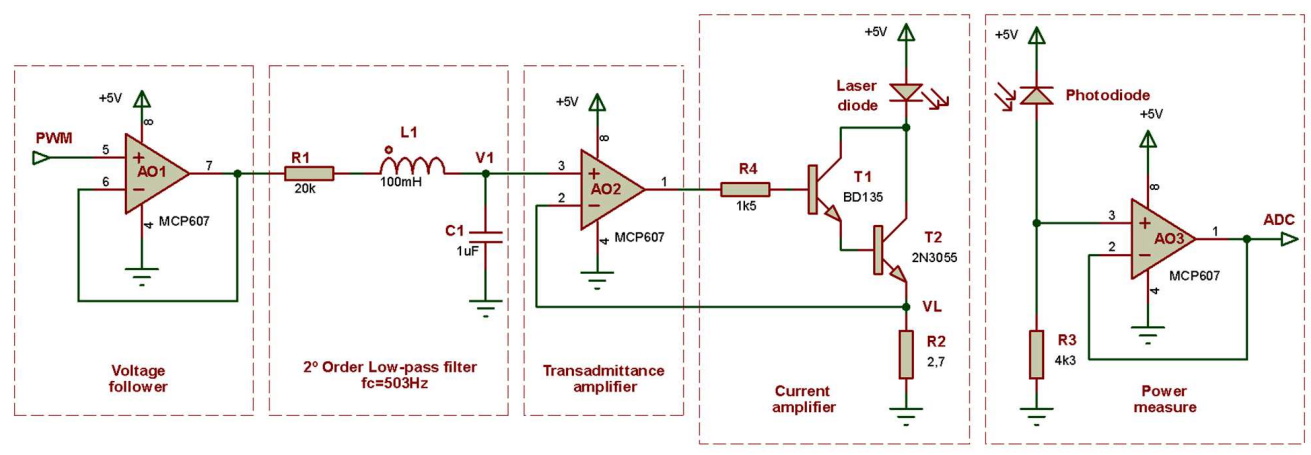

Figure 5: Voltage-to-current converter including voltage follower, low-pass filter and amplifiers. The circuit including the photodiode, on the right, provides feedback to the controller.

The core element of both the voltage follower and the transmittance amplifier is a linear operational amplifier (OA) (MCP607, manufactured by Microchip Technology Inc). This is a rail-to-rail OA based on CMOS technology. Its high input impedance (in the range of $T \Omega$ ) and large operating bandwidth (over $100 \mathrm{kHz}$ ) make it appropriate for this application. The cut-off frequency of the RLC low-pass filter equals $503 \mathrm{~Hz}$.

The current amplifier is mainly composed by two bipolar junction transistors set up as a Darlington pair operating in linear conditions. The rationale for choosing this design for the group formed by the transadmittance and current amplifiers is as follows. The high input impedance of the MCP607 OA isolates the amplifier input from the output of the RLC filter. This avoids current leakage from the filter to the OA and minimises the ripple at the filter's output. Additionally, the high input impedance makes the voltage at both the positive and the negative inputs of the $\mathrm{OA}$ almost equal, i.e. $V_{1}$. The output current of the OA feeds the Darlington pair which, in turn, causes a voltage $V_{\mathrm{L}}$ on resistor $R_{2}$. The negative feedback of the OA forces $V_{\mathrm{L}}=V_{1}$, so the current at the laser diode is made proportional to $V_{1}$, being $R_{2}$, the proportionality factor. Its value, that of $R_{2}$ is chosen so that the OA works in linear conditions for all the current range of the laser diode.

The rightmost part of the electronic schema in Figure 5 is a circuit containing a photodiode and an OA. The photodiode is packaged with the laser diode [28]. On receiving the laser radiation, a current crosses the photodiode that causes a voltage across $R_{3}$. This voltage signal, after amplification by an $\mathrm{OA}$, is sent to the analogue input of the micro-controller as a feedback 
signal.

\subsubsection{Controller and user interface}

As previously mentioned, the laser controller mainly consists of a 8-bit micro-controller and a user interface (see Figure 1) that includes a liquid crystal display (LCD) and three buttons that allow the user to navigate through an options menu. The user can choose among two working modes for the laser emission: continuous or pulsed. For each mode, the user can also choose among 101 possible values for the radiated power.

The firmware of the system is aimed at providing a user-friendly interface. As mentioned above, this interface is based on a menu system, which is illustrated by the flow diagram in Figure 6. The structure of the menu system basically comprises two distinct parts: operations menu and settings menu. The operations menu allows starting the experiment. While the experiment runs, the LCD display shows the elapsed fraction of the experiment duration and the power estimated by the measuring circuit (rightmost circuit in Figure 5). This allows the user to check whether the system is delivering the requested power. An error checking routine has also been implemented that alerts the user via the LCD display in case some anomaly happens while the laser actuator is working.

The settings menu provides options for configuring three different aspects of the experiment: time, current and alarm. Time configuration includes setting the overall duration of the experiment, from 1 second to 60 minutes, the working mode, the pulse period and the pulse length. As mentioned before, the user can choose the current intensity for the laser diode among 40 possible values. Last, a buzzing alarm can be activated that informs the user about the end of the experiment. All configurable parameters related to time, current and alarm are stored in a set of 10 internal memories.

\subsubsection{Transfer function}

The microcontroller includes a CCP module (capture, compare and PWM) that allows it to control the width of the output voltage pulses. This width, that is, the working cycle of the PWM signal, is proportional to the current intensity at the laser diode. The word length of the microcontroller ( 8 bits) allows 256 different values for the PWM working cycle $\left(N_{\text {res }}=256\right)$. However, in order to maintain the current at the laser diode inside the range corresponding to linear operation conditions, the user is only allowed to choose among 101 possible values. 


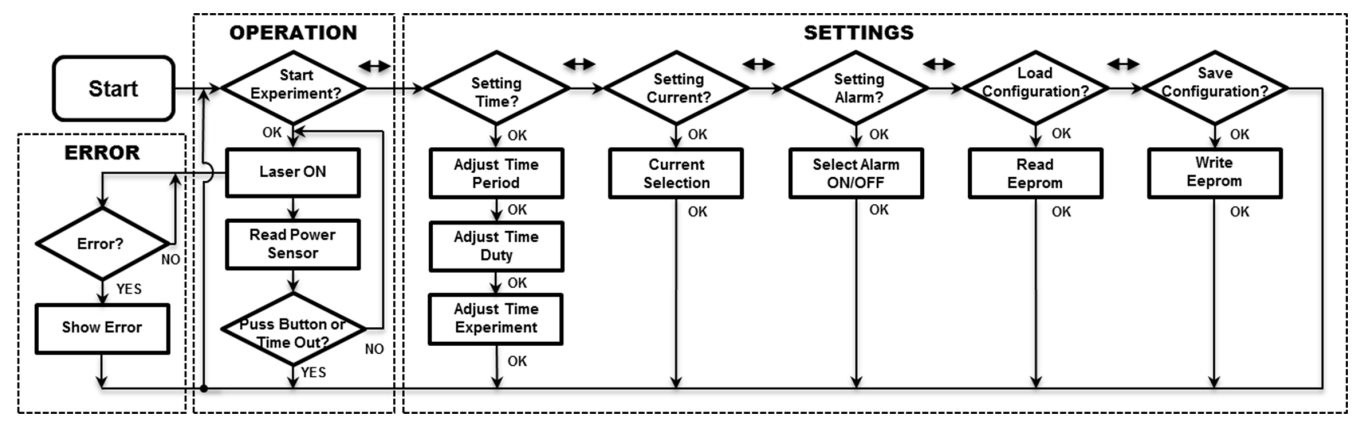

Figure 6: Structure of the options menu in the user interface.

The voltage-to-current converter (Figure 5) has the following transfer function:

$$
I_{\mathrm{ld}}=\frac{V_{1}}{R_{2}}
$$

where $I_{\text {ld }}$ is the current at the laser diode. $R_{2}$ was chosen so that the maximum current potentially delivered by the converter to the laser diode was above its nominal value $I_{\max }>500 \mathrm{~mA}$. Specifically, for $R_{2}=2.7 \Omega$ it results $I_{\max }=1.1 \mathrm{~A}$. By operating the controls of the power system, the user can choose the actual value for $I_{\mathrm{ld}}$ as:

$$
I_{\mathrm{ld}}=\frac{V_{1}}{R_{2}}=n \frac{I_{\mathrm{max}}}{N_{\mathrm{res}}}, \quad 1 \leq n \leq N_{\mathrm{res}}
$$

where $n$ represents the user selection. In principle, $n$ could vary from 1 to $N_{\text {res }}$. However, in order to keep the working conditions of the laser diode within the range corresponding to linear behaviour (see Figure 7) user selection was limited to $46 \leq n \leq 146$, which correspond to current $I_{\mathrm{ld}}$ varying between $198 \mathrm{~mA}$ and $627 \mathrm{~mA}$. For this range of current intensities, the powerto-current relation for the laser diode can be assumed to be linear, according to the following equation:

$$
P_{\mathrm{ld}}[\mathrm{W}] \approx 1.07 I_{\mathrm{ld}}[\mathrm{A}]-0.2
$$

Therefore, the relation between laser power and user selection it can be approximated as:

$$
P_{\mathrm{ld}}[\mathrm{W}] \approx \frac{1.07 n I_{\max }[\mathrm{A}]}{N_{\mathrm{res}}}-0.2
$$




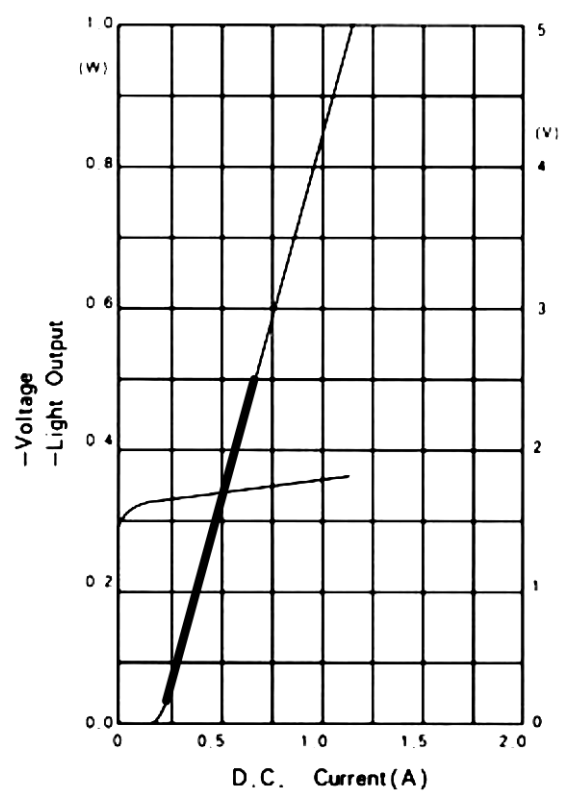

Figure 7: Voltage-to-current and power-to-current graphs for the laser diode (taken from [28]). The thicker straight segment indicates the working conditions for the proposed system.

Considering (3), the radiated power density as a function of user selection is:

$$
L\left[\mathrm{~W} / \mathrm{cm}^{2}\right] \approx \frac{1}{\pi a[\mathrm{~cm}] b[\mathrm{~cm}]}\left(\frac{1.07 n I_{\max }[\mathrm{A}]}{N_{\mathrm{res}}}-0.2\right)
$$

where $a$ and $b$ are measured in the calibration stage. For the experiments reported in Section 3, if $I_{\mathrm{ld}}$ was set to $627 \mathrm{~mA}(n=146)$ then the obtained radiated power density was $L=4 \mathrm{~W} / \mathrm{cm}^{2}$; for $n=46\left(I_{\mathrm{ld}}=198 \mathrm{~mA}\right)$ the radiated power density was $0.098 \mathrm{~W} / \mathrm{cm}^{2}$.

\subsection{Thermostatic chamber}

Figure 8 provides an overview of the thermostatic chamber. Its outer case is made of transparent polycarbonate plastic. This allows visual supervision from the outside and it helps in adequately positioning all elements before starting any experiment. In addition to the laser diode and the power system, these elements include:

- The digital microscope, connected to a PC. It not only helps in calculating the irradiated surface, as explained before, but also in placing 
the sample to be irradiated into the laser beam.

- A plastic support for a 96-well ELISA microplate. ELISA microplates are of common use for cell culture, and they are herein utilised for containing the irradiated samples. The multiple wells of the microplate make it possible to work with diverse samples within a single experiment. The position of the plastic support is adjustable so that the operator can align the laser beam and the sample well.

- A temperature probe that allows supervision of the temperature in the processed sample well. For the experiments reported in next section, an optic-fibre probe was used, with the fibre coated with heat-shrink tubing in order to avoid interference from the laser beam. Thermocouples provide a cheaper alternative which can also be supervised by a microcontroller in case a higher degree of automation is desired.

The chamber is equipped with an air-heating system that allows running the experiment at temperatures higher than that of the environment. This air-heating system mainly consists in a series connection of two power resistors. The resistors are linked to metallic heat sinks that ease the transfer of heat to the air. The resistors are placed in transverse position into a nozzle inside which air circulates as a result of the action of an electric fan. Air circulates downwards inside the nozzle and upwards inside the chamber.

The functioning of the air-heating system is controlled by another microcontroller (also a PIC18F2550) that receives data from a digital thermometer (DS1631, manufactured by Maxim Integrated) placed at the top of the nozzle. Thus, it measures the air temperature at the input of the air-heating system. Registered temperature data are sent to the micro-controller through a I2C bus. The micro-controller acts on both the resistors and the fan with an intermediate amplifying stage acting as interface.

The target temperature inside the chamber can be adjusted in the $30^{\circ} \mathrm{C}$ to $40^{\circ} \mathrm{C}$ range by operating a potentiometer accessible from the outside. A $2 \times 16$ LCD display serves as a user interface that allows the human operator to supervise the temperature inside the chamber. Alternatively, the microcontroller can be connected to a PC using a series-to-USB adapter in order to make it possible a more complex processing of data, such as generating graphic plots. The PC also allows issuing instructions to the controller by means of a command line interface. 


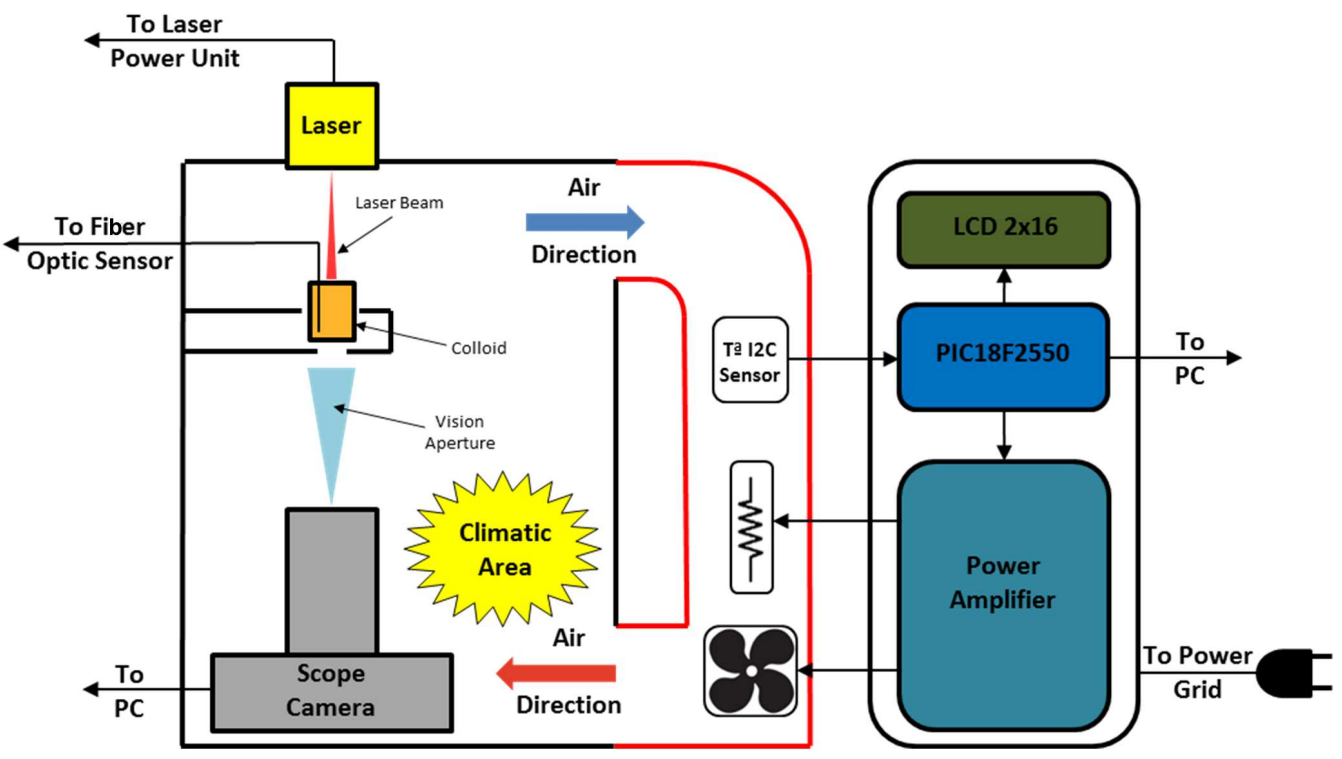

Figure 8: Schematic diagram on the thermostatic chamber.

The firmware of the temperature controlling system aims at achieving the target temperature in the shortest possible time. For this purpose, it implements a proportional-integral (PI) controller that actuates the resistors and a multilevel controller that actuates the fan. Actuation of the resistors is achieved by making use of one of the PWM outputs of the controller. The PWM voltage reduces its working cycle as the temperature approaches its target value. As for the fan, its three-level regulation is controlled by another PWM output of the controller. In this case, transitions between levels are controlled by a hysteresis algorithm with adjustable temperature thresholds. Fan speed is an increasing function of the absolute difference between actual and target chamber temperatures.

\section{Experiments and results}

Two different experiments were carried out in order to test the potential applicability of the developed equipment. A different kind of $\mathrm{Au}-\mathrm{NP}$ was used for each experiment: nanorods for the first one and nanostars for the second. In both experiments, the radiated power density was variable along time and the temperature evolution of the irradiated $\mathrm{Au}-\mathrm{NP}$ suspensions was measured. These two data sequences, radiated power density and tempera- 
ture, were used for estimating the specific absorption rate (SAR), which is a straightforward way of evaluating the efficiency of the equipment.

\subsection{First experiment: nanorods}

\subsubsection{Synthesis of gold nanorods}

Gold nanorods absorbing light with wavelengths in the $700-800 \mathrm{~nm}$ range were synthesised for this experiment. Synthesis was realised according to El-Sayed method [12, 29, 30]:

1. The growth solution was prepared at $27^{\circ} \mathrm{C} .5 \mathrm{ml}$ of a $10 \mathrm{mM}$ solution of $\mathrm{HAuCl}_{4}$ were added to $5 \mathrm{ml}$ of cetyltrimethylammonium bromide (CTAB).

2. Afterwards, $250 \mu \mathrm{l}$ of a $4 \mathrm{mM}$ solution of $\mathrm{AgNO}_{3}$ were added and the solution was stirred.

3. Next, a $\mathrm{HCl}$ solution at $37 \%$ concentration was added to obtain a $\mathrm{pH}$ value between 1 and 1.15 .

4. $70 \mu \mathrm{l}$ of $78.8 \mathrm{mM}$ ascorbic acid were subsequently added to the solution until it became colourless.

5. Immediately after that, $15 \mu \mathrm{l}$ of an ice-cold $10 \mathrm{mM}$ solution of $\mathrm{NaBH}_{4}$ were added and allowed to react during 6 hours.

6. Last, the resulting solution was spun during 15 minutes at 14,500 rpm. The supernatant was removed and the precipitate was mixed in water and spun again at the same speed and during the same time.

$\mathrm{HAuCl}_{4}, \mathrm{AgNO}_{3}, \mathrm{NaBH}_{4}$, ascorbic acid, and CTAB were purchased from Sigma-Aldrich. $\mathrm{HCl}$ was purchased from Sharlab.

\subsubsection{Characterization of synthesised gold nanorods}

The shape and size of the synthesised gold nanorods were characterized both by surface plasmon resonance spectroscopy and by transmission electron microscopy (TEM). The length of the nanorods was $25 \mathrm{~nm}$ and their diameter was $6 \mathrm{~nm}$. All spectrophotometric studies were carried out using a V-630 UVVIS spectrophotometer, manufactured by Jasco Deutschland GmbH. $1 \mathrm{ml}$ of the suspension containing the gold nanorods was thoroughly sonicated for five minutes and then the absorbance spectrum was measured, taking water as a reference. The measured absorbance spectrum is plotted in Figure 9. Two surface plasmon bands can be noticed at $520 \mathrm{~nm}$ and $800 \mathrm{~nm}$.

TEM measurements were performed using a JEM-1010 microscope, manufactured by JEOL Ltd. Samples were prepared by placing small drops of the 


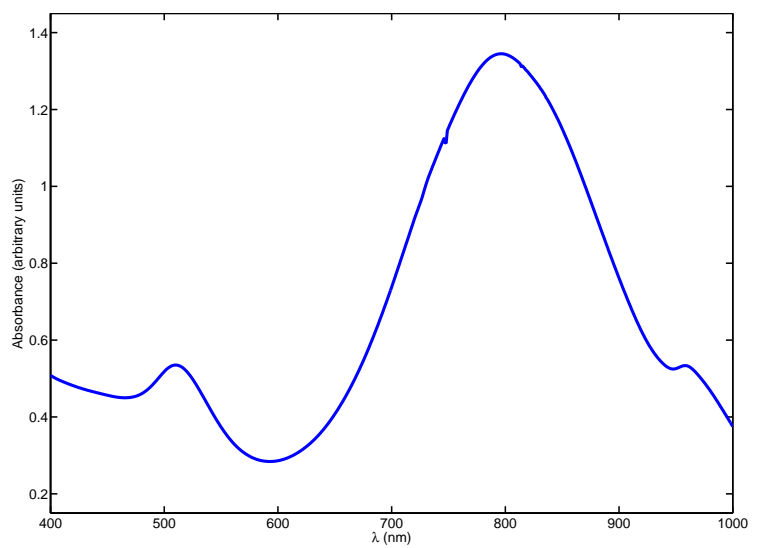

Figure 9: Absorbance spectrum of nanorods measured using spectrophotometry.

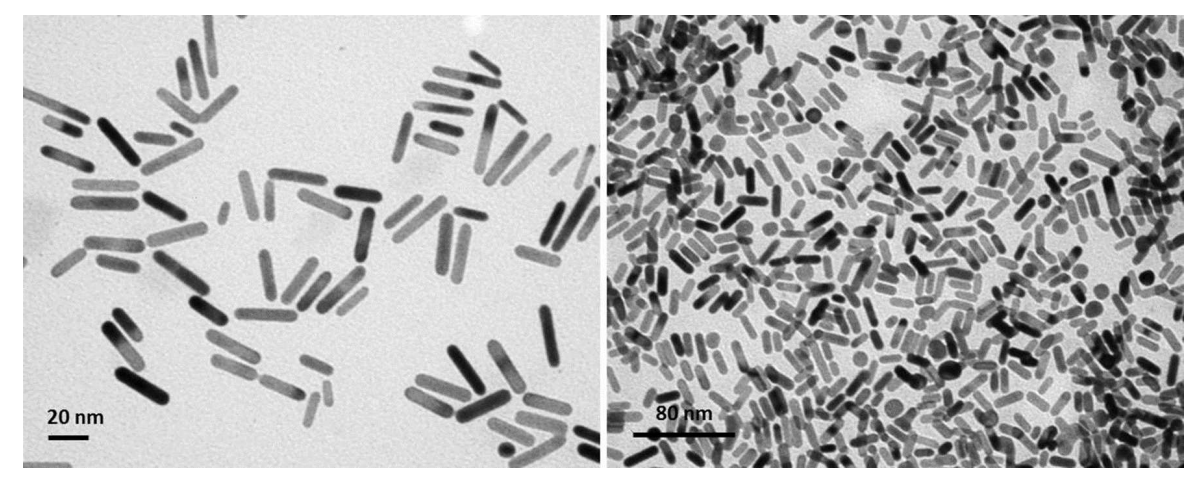

Figure 10: TEM images of synthesised nanorods at two different scales.

suspension containing the gold nanorods onto formvar-coated copper grids and by subsequently allowing the solvent (water) to evaporate slowly at room temperature. The dried grid was then examined under the transmission electron microscope. Two images from the gold nanorods generated using TEM are depicted in Figure 10.

\subsubsection{Laser induced heating of gold nanorods}

Approximately $600 \mu \mathrm{l}$ of gold nanorod solution were obtained as a result of the afore-mentioned synthesis process. This volume was split into three equal parts (200 $\mu \mathrm{l}$ each), and each part was subsequently poured into a different well in a microplate. The concentration of the solution was estimated by measuring the extinction spectrum at $400 \mathrm{~nm}$ (as in [31, 32]), resulting $38.4 \mu \mathrm{g} / \mathrm{ml}$. 

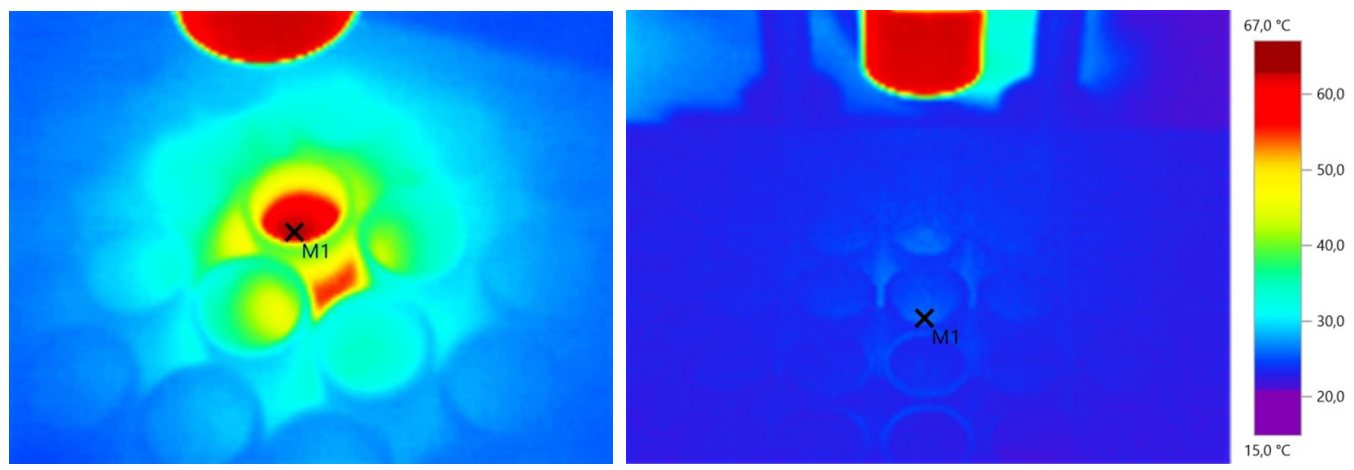

Figure 11: Thermographic images of the microplate obtained with the Testo 875 camera. The cross (M1) indicates the hottest point on the microplate surface: $64.6^{\circ} \mathrm{C}$ for the nanorod solution (left) and $25.6^{\circ} \mathrm{C}$ for water (right).

The laser diode was placed $1 \mathrm{~cm}$ above the sample well to be heated. This implied that approximately $30.6 \%$ of the surface of the nanorod solution was irradiated by the laser beam. Each sample underwent five radiation intervals, each one lasting for 15 minutes and followed by a 4-minute cooling period. The power system was calibrated to deliver a distinct power density for each interval, namely $0.1,1,2,3$ and $4 \mathrm{~W} / \mathrm{cm}^{2}$.

During each radiation plus cooling interval, the temperature of the colloidal suspension was supervised using a thermographic camera (Testo 875, manufactured by Testo $A G$ ) that captured one image every 20 seconds. For each image (Figure 11), the hottest point corresponding to the microplate surface was obtained, with its corresponding temperature. Temperature values for each time instant and each radiated power were averaged for the three parts of the solution. These average values are plotted in Figure 12. The highest temperature was reached at $4 \mathrm{~W} / \mathrm{cm}^{2}$ radiated power after $10 \mathrm{~min}$ utes of irradiation. Note that temperature increments approximately ranged from $10^{\circ} \mathrm{C}$ to $40^{\circ} \mathrm{C}$, depending on the delivered radiation power. In a similar experiment carried out substituting the $\mathrm{Au}-\mathrm{NP}$ suspension with distilled water the temperature increment hardly reached $2^{\circ} \mathrm{C}$. This implies that the highest achieved temperature was $39.2^{\circ} \mathrm{C}$ above the temperature registered for water in the same conditions. 


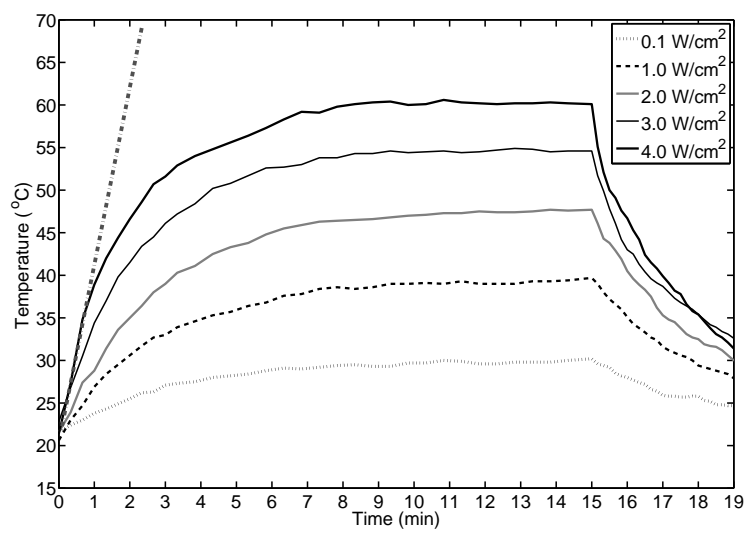

Figure 12: Average temperature in the nanorod solution as a function of time and radiated power. The straight line approximately indicates the initial slope of the curve corresponding to $4 \mathrm{~W} / \mathrm{cm}^{2}$.

\subsection{Second experiment: nanostars}

\subsubsection{Synthesis of gold nanostars}

Gold nanostars were synthesized by a seeded growth method using polyvinylpyrrolidone $(\mathrm{PVP}$, molecular weight $=10,000)$ and N,N-dimethylformamide $(\mathrm{DMF})$ [33] with minor modifications:

1. $50 \mu \mathrm{l}$ of a $166 \mathrm{mM}$ aqueous solution of $\mathrm{HAuCl}_{4}$ were mixed with $15 \mathrm{ml}$ of a $20 \mathrm{mM}$ solution of PVP in DMF at $25^{\circ} \mathrm{C}$.

2. 5 minutes later, $10 \mu \mathrm{l}$ of a pre-formed $6.5 \mathrm{mM}$ seed dispersion $(15 \mathrm{~nm}$ gold nanospheres coated with PVP in ethanol) were added and let react for 12 hours without stirring.

3. After reaction, Au-NPs were recovered by centrifugation at 9,500 rpm during 20 minutes.

4. Recovered Au-NPs were washed in water five times, by redispersion and centrifugation.

\subsubsection{Characterisation of synthesised gold nanostars}

The absorbtion spectrum of gold nanostars (Figure 13) shows two surface plasmon bands at $560 \mathrm{~nm}$ and $796 \mathrm{~nm}$, respectively. The first one corresponds to localized surface plasmon resonance (LSPR) of core electrons, while the second one is associated to localized surface plasmon resonance of tip electrons [33]. As for their size, the obtained gold nanostars have a 40-nm core with multiple sharp branches of lengths around $25 \mathrm{~nm}$ (Figure 14). 


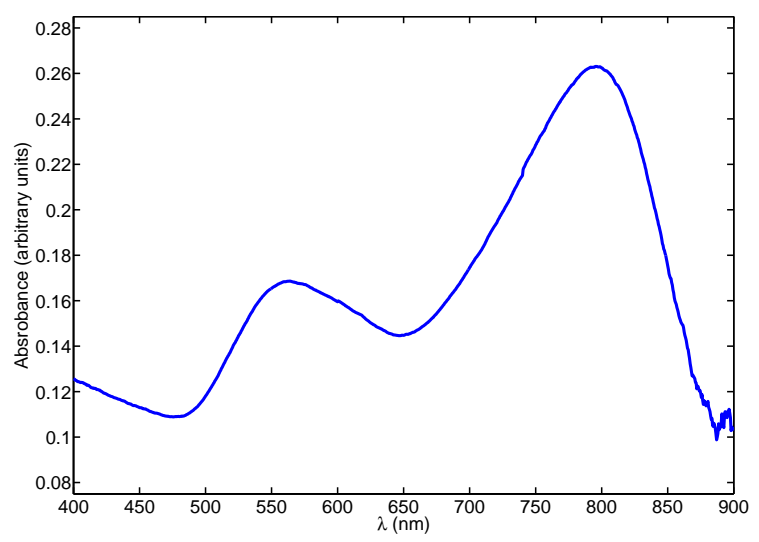

Figure 13: Absorbance spectrum of nanostars measured using spectrophotometry.

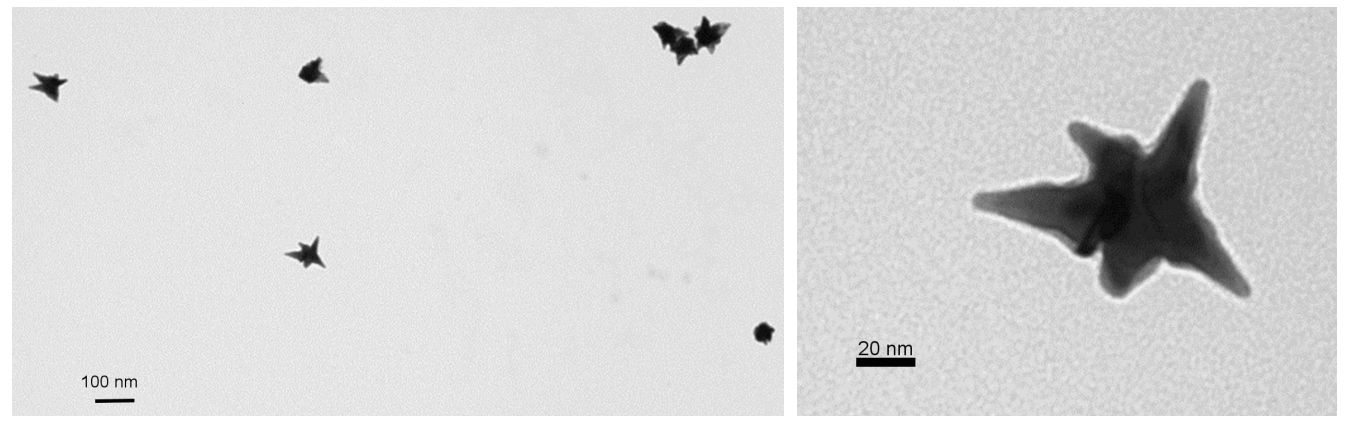

Figure 14: TEM images of synthesised nanostars at two different scales.

\subsubsection{Laser induced heating of gold nanostars}

The laser induced heating experiments for gold nanostars were performed with the same system and diode-to-sample distance as for nanorods. In this case, each well in the micro-plate was filled with $250 \mu \mathrm{l}$ of nanostar supension with concentration equal to $188 \mu \mathrm{g} / \mathrm{ml}$. The air inside the thermostatic chamber was forced to be at $37^{\circ} \mathrm{C}$. The evolution of temperature in the irradiated samples was monitored using a fiber-optic thermosensor (Figure $15)$.

\section{3. $S A R$ vs radiated power density}

In order to have a more complete insight into the performance of the developed equipment, the SAR has been evaluated as a function of radiated power density. The SAR is defined as the ratio between absorbed power and 


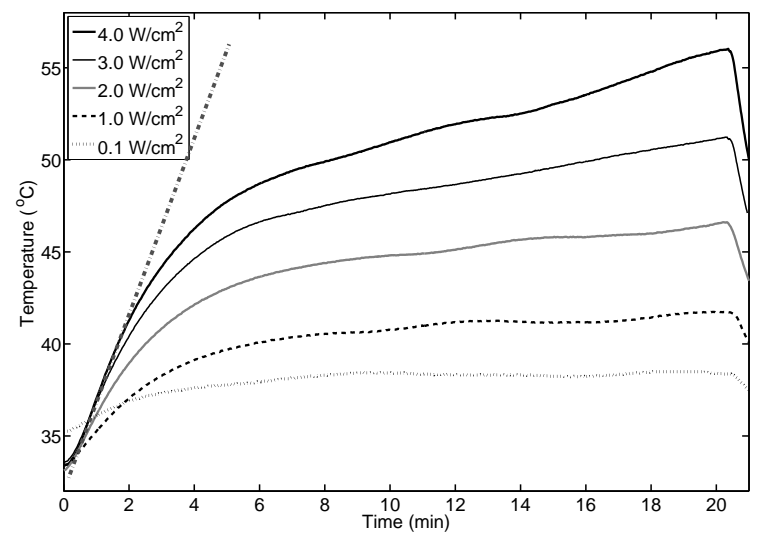

Figure 15: Average temperature in the nanostar solution as a function of time and radiated power. The straight line approximately indicates the initial slope of the curve corresponding to $4 \mathrm{~W} / \mathrm{cm}^{2}$.

mass of the irradiated sample. The SAR can be estimated as $[7,9,34]$

$$
S A R=\frac{c_{\mathrm{s}} \rho_{\mathrm{s}} V_{\mathrm{s}}}{m_{\mathrm{Au}}} \cdot \frac{\Delta T}{\Delta t}
$$

where $c$ is specific heat, $\rho$ is mass density, $V$ is its volume, $m$ is the mass, the subscript s refers to the suspension of nanoparticles, the subscript Au refers to the suspended $\mathrm{Au}-\mathrm{NPs}$, and $\frac{\Delta T}{\Delta t}$ is the initial slope of the temperature versus time curves in Figures 12 and 15. $m_{\mathrm{Au}}$ was measured immediately after synthesising the Au-NPs, while the values for $\rho_{\mathrm{s}}$ and $V_{\mathrm{s}}$ have already been mentioned. In turn, $c_{\mathrm{s}}$ was estimated as [35]:

$$
c_{\mathrm{s}} \approx \frac{\phi \rho_{\mathrm{Au}} c_{\mathrm{Au}}+(1-\phi) \rho_{\mathrm{H}_{2} \mathrm{O}} c_{\mathrm{H}_{2} \mathrm{O}}}{\phi \rho_{\mathrm{Au}}+(1-\phi) \rho_{\mathrm{H}_{2} \mathrm{O}}}
$$

where $\phi$ is the fraction of the suspension volume occupied by the Au-NPs, and the subscript $\mathrm{H}_{2} \mathrm{O}$ refers to the solvent, i.e. water. Figure 16 shows that the relation between SAR and radiated power density is fairly linear. There also is a liner relation between the radiated power density $L$ and user power selection $n$ (8). Thus, the experimental data indicate that for this equipment the user can linearly control the SAR.

\section{Conclusion}

This paper introduces an electronic system aimed at experimenting with laser applications such as controlled drug release or optical hyperthermia. 


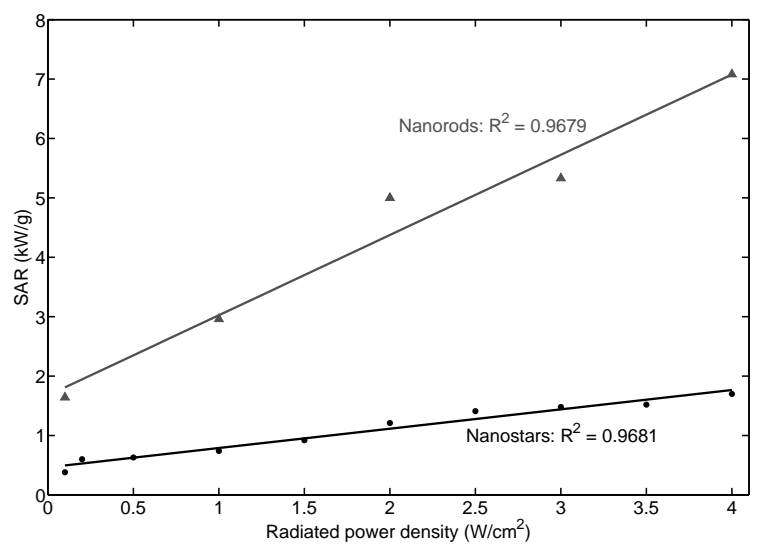

Figure 16: Estimated SAR versus radiated power density. Linear regression yields a determination coefficient equal to 0.9679 for nanorods and 0.9681 for nanostars.

The design of the system has been carried out pursuing two main objectives: low cost and flexible configuration. Low cost has been achieved by tuning the characteristics of the system to the specific target applications, hence avoiding superfluous subsystems. The design of the electronic components allow the user to configure parameters such as output power, type of laser emission (continuous vs pulsed) or irradiated area. Specifically, the irradiated area can be supervised thanks to the inclusion of an optical system. Last, setting up the equipment into a polycarbonate chamber allows controlling both the precise positions of all elements and the ambient temperature.

The experiments performed with testing purposes have been successful in that they have shown that the equipment fulfils its purpose of transferring light energy to Au-NPs suspended in a solvent without directly heating the solvent (distilled water). Namely, a suspension of gold nanorods has been heated up to $64.6^{\circ} \mathrm{C}$ from an ambient temperature equal near $23^{\circ} \mathrm{C}$ (Figure 12) while the solvent alone, without suspended Au-NPs, only reached $25.6^{\circ} \mathrm{C}$ in the same conditions. A similar experiment, with the same radiated power density $\left(4 \mathrm{~W} / \mathrm{cm}^{2}\right)$, was performed with gold nanostars and changing the ambient temperature to a value similar to that of the human body $\left(37^{\circ}\right)$. In this case, the solution reached $50.6^{\circ} \mathrm{C}$ after 10 minutes of exposure to laser radiation (Figure 15).

The SAR is a magnitude typically used for assessing hyperthermia treatments. The evaluated prototype has reached SAR values in the range of 1.7 $\mathrm{kW} / \mathrm{g}$ for gold nanostars (Figure 16), which is a high enough value for optical 
hyperthermia [36]. Higher values were obtained for nanorods (between 2 and $7 \mathrm{~kW} / \mathrm{g}$ ). Additionally, in both cases the SAR almost is a linear function of the user-selected value for the current intensity at the laser diode, which is also linearly related to the radiated power. This fact is important in what implies easiness of regulation.

Last, it is worth noting that the fact that the inclusion of microcontrollers for supervising the ambient conditions of the experiment, together with the possibility of connecting them to external computers, makes it possible to evolve the system to include more complex control functions in the future, such as automatic control of the radiated power density or closed-loop temperature control using thermographic cameras [37].

\section{Acknowledgements}

This work has been carried out in the context of project "Development of electronic devices and equipment for detection and actuation based on new electronic technologies. Applications in the field of biomedicine" (grant number: MAT2015-64139-C4-3-R) and in coordination with project number MAT2015-64139-C4-1-R, both of them financed by the Spanish Ministry for Economy and Competitiveness in the framework of the Plan Estatal de Investigación Científica y Técnica y de Innovación 2013-2016. Financial support from the Generalitat Valenciana (PROMETEOII/2014/047) and from the UPV (Erasmus Mundus EuroInkaNet project, 2013 call) are gratefully acknowledged too.

\section{References}

[1] R. S. Ningthoujam, R. K. Vatsa, A. Kumar, B. N. Pandey, Functionalized magnetic nanoparticles: Concepts, synthesis and application in cancer hyperthermia, in: S. Banerjee, A. K. Tyagi (Eds.), Functional Materials: Preparation, Processing and Applications, Elsevier, London, 2012, Ch. 6, pp. 229-260.

[2] J. L. Li, M. Gu, Gold-nanoparticle-enhanced cancer photothermal therapy, IEEE Journal of Selected Topics in Quantum Electronics 16 (4) (2010) 989-996.

[3] X. Huang, I. H. El-Sayed, W. Qian, M. A. El-Sayed, Cancer cell imaging and photothermal therapy in the near-infrared region by using gold 
nanorods, Journal of the American Chemical Society 128 (6) (2006) 2115-2120.

[4] P. K. Jain, X. Huang, I. H. El-Sayed, M. A. El-Sayed, Noble metals on the nanoscale: Optical and photothermal properties and some applications in imaging, sensing, biology, and medicine, Accounts of Chemical Research 41 (12) (2008) 1578-1586.

[5] Y. Liu, H. Yuan, A. M. Fales, J. K. Register, T. Vo-Dinh, Multifunctional gold nanostars for molecular imaging and cancer therapy, Frontiers in Chemistry 3 (Article ID 51) (2015) 1-7.

[6] S. Harmsen, R. Huang, M. A. Wall, H. Karabeber, J. M. Samii, M. Spaliviero, J. R. White, S. Monette, R. OConnor, K. L. Pitter, S. A. Sastra, M. Saborowski, E. C. Holland, S. Singer, K. P. Olive, S. W. Lowe, R. G. Blasberg, M. F. Kircher, Surface-enhanced resonance Raman scattering nanostars for high-precision cancer imaging, Science Translational Medicine 7 (271) (2015) 271ra7.

[7] A. M. Fales, H. Yuan, T. Vo-Dinh, Cell-penetrating peptide enhanced intracellular Raman imaging and photodynamic therapy, Molecular Pharmaceutics 10 (6) (2013) 2291-2298.

[8] P. K. Jain, I. H. El-Sayed, M. A. El-Sayed, Au nanoparticles target cancer, Nano Today 2 (1) (2007) 18-29.

[9] P. del Pino, B. Pelaz, Hyperthermia using inorganic nanoparticles, in: J. M. de la Fuente, V. Grazo (Eds.), Nanobiotechnology: Inorganic Nanoparticles vs Organic Nanoparticles, Vol. 4 of Frontiers in Nanoscience, Elsevier, Oxford, 2012, Ch. 13, pp. 309-335.

[10] P. C. Chen, S. C. Mwakwari, A. K. Oyelere, Gold nanoparticles: From nanomedicine to nanosensing, Nanotechnology, Science and Applications 1 (2008) 45-66.

[11] K. W. Hu, T. M. Liu, K. Y. Chung, K. S. Huang, C. T. Hsieh, C. K. Sun, C. S. Yeh, Efficient near-IR hyperthermia and intense nonlinear optical imaging contrast on the gold nanorod-in-shell nanostructures, Journal of the American Chemical Society 131 (40) (2009) 14186-14187. 
[12] J. Pérez-Juste, I. Pastoriza-Santos, L. M. Liz-Marzán, P. Mulvaney, Gold nanorods: Synthesis, characterization and applications, Coordination Chemistry Reviews 249 (17) (2005) 1870-1901.

[13] H. C. Huang, S. Barua, D. B. Kay, K. Rege, Simultaneous enhancement of photothermal stability and gene delivery efficacy of gold nanorods using polyelectrolytes, ACS Nano 3 (10) (2009) 2941-2952.

[14] L. M. Maestro, P. Haro-González, A. Sánchez-Iglesias, L. M. LizMarzán, J. García Solé, D. Jaque, Quantum dot thermometry evaluation of geometry dependent heating efficiency in gold nanoparticles, Langmuir 30 (6) (2014) 1650-1658.

[15] R. Rodríguez-Oliveros, J. A. Sánchez-Gil, Gold nanostars as thermoplasmonic nanoparticles for optical heating, Optics Express 20 (1) (2012) $621-626$.

[16] A. Jordan, R. Scholz, P. Wust, H. Fähling, R. Felix, Magnetic fluid hyperthermia $(\mathrm{MFH})$ : Cancer treatment with $\mathrm{AC}$ magnetic field induced excitation of biocompatible superparamagnetic nanoparticles, Journal of Magnetism and Magnetic Materials 201 (1) (1999) 413-419.

[17] G. C. Li, N. F. Mivechi, G. Weitzel, Heat shock proteins, thermotolerance, and their relevance to clinical hyperthermia, International Journal of Hyperthermia 11 (4) (1995) 459-488.

[18] A. Chicheł, J. Skowronek, M. Kubaszewska, M. Kanikowski, Hyperthermia-description of a method and a review of clinical applications, Reports of Practical Oncology \& Radiotherapy 12 (5) (2007) $267-275$.

[19] V. S. Kalambur, E. K. Longmire, J. C. Bischof, Cellular level loading and heating of superparamagnetic iron oxide nanoparticles, Langmuir 23 (24) (2007) 12329-12336.

[20] P. Cherukuri, E. S. Glazer, S. A. Curley, Targeted hyperthermia using metal nanoparticles, Advanced Drug Delivery Reviews 62 (3) (2010) 339-345.

[21] N. Khlebtsov, V. Bogatyrev, L. Dykman, B. Khlebtsov, S. Staroverov, A. Shirokov, L. Matora, V. Khanadeev, T. Pylaev, N. Tsyganova, 
G. Terentyuk, Analytical and theranostic applications of gold nanoparticles and multifunctional nanocomposites, Theranostics 3 (3) (2013) $167-80$.

[22] R. Bhattacharya, P. Mukherjee, Biological properties of "naked" metal nanoparticles, Advanced Drug delivery Reviews 60 (11) (2008) 12891306.

[23] R. Hong, G. Han, J. M. Fernández, B. Kim, N. S. Forbes, V. M. Rotello, Glutathione-mediated delivery and release using monolayer protected nanoparticle carriers, Journal of the American Chemical Society 128 (4) (2006) 1078-1079.

[24] S. Al-Qadi, C. Remuñán-López, Nanopartículas metálicas: Oro, in: J. L. Vila (Ed.), Nanotecnología Farmacéutica: Realidades y Posibilidades Farmacoterapéuticas, Vol. XXVIII of Monografías de la Real Academia Nacional de Farmacia, Real Academia Nacional de Farmacia, Madrid, 2009, Ch. 7, pp. 223-248.

[25] G. Han, C. T. Martin, V. M. Rotello, Stability of gold nanoparticlebound DNA toward biological, physical, and chemical agents, Chemical Biology \& Drug Design 67 (1) (2006) 78-82.

[26] G. Han, C. C. You, B. Kim, R. S. Turingan, N. S. Forbes, C. T. Martin, V. M. Rotello, Light-regulated release of DNA and its delivery to nuclei by means of photolabile gold nanoparticles, Angewandte Chemie 118 (19) (2006) 3237-3241.

[27] A. J. Gormley, K. Greish, A. Ray, R. Robinson, J. A. Gustafson, H. Ghandehari, Gold nanorod mediated plasmonic photothermal therapy: A tool to enhance macromolecular delivery, International Journal of Pharmaceutics 415 (1) (2011) 315-318.

[28] LDM-0808-500m-92, Technical data, Roithner LaserTechnik (2010). URL http://www.roithner-laser.com/datasheets/ld_div/ 1dm_0808_500m_92.pdf [Visited: 25/06/2016]

[29] M. R. K. Ali, B. Snyder, M. A. El-Sayed, Synthesis and optical properties of small Au nanorods using a seedless growth technique, Langmuir 28 (25) (2012) 9807-9815. 
[30] E. Leontidis, K. Kleitou, T. Kyprianidou-Leodidou, V. Bekiari, P. Lianos, Gold colloids from cationic surfactant solutions. 1. Mechanisms that control particle morphology, Langmuir 18 (9) (2002) 36593668 .

[31] J. Rodríguez-Fernández, J. Pérez-Juste, P. Mulvaney, L. M. Liz-Marzán, Spatially-directed oxidation of gold nanoparticles by $\mathrm{Au}$ (III)-CTAB complexes, Journal of Physical Chemistry B 109 (30) (2005) 1425714261.

[32] L. Scarabelli, M. Grzelczak, L. M. Liz-Marzán, Tuning gold nanorod synthesis through prereduction with salicylic acid, Chemistry of Materials 25 (21) (2013) 4232-4238.

[33] A. Guerrero-Martínez, S. Barbosa, I. Pastoriza-Santos, L. M. LizMarzán, Nanostars shine bright for you: Colloidal synthesis, properties and applications of branched metallic nanoparticles, Current Opinion in Colloid \& Interface Science 16 (2) (2011) 118-127.

[34] G. Vallejo-Fernández, O. Whear, A. G. Roca, S. Hussain, J. Timmis, V. Patel, K. O'Grady, Mechanisms of hyperthermia in magnetic nanoparticles, Journal of Physics D: Applied Physics 46 (31) (2013) 312001 .

[35] J. Buongiorno, Convective transport in nanofluids, Journal of Heat Transfer 128 (3) (2006) 240-250.

[36] J. H. Lee, J. Jang, J. Choi, S. H. Moon, S. Noh, J. Kim, J. G. Kim, I. S. Kim, K. I. Park, J. Cheon, Exchange-coupled magnetic nanoparticles for efficient heat induction, Nature Nanotechnology 6 (7) (2011) 418-422.

[37] D. P. O’Neal, L. R. Hirsch, N. J. Halas, J. D. Payne, J. L. West, Photothermal tumor ablation in mice using near infrared-absorbing nanoparticles, Cancer Letters 209 (2) (2004) 171-176. 